\title{
THE QUALITATIVE EXAMINATION OF URINARY CALCULI
}

\author{
By JOHN F. MCINTOSH AND RUTH W. SALTER \\ (From the Royal Victoria Hospital and the McGill University Clinic, Montreal)
}

(Received for publication May 25, 1942)

Heller's (1) monograph, published in 1860, contains a scheme for the chemical examination of urinary calculi which has succeeded in holding the field for 80 years. This scheme is still reproduced in standard textbooks and in general use in many clinics, if we may judge from the periodic literature $(2,3)$.

Heller and his successors depended largely upon the use of mechanical means to separate the different constituents of calculi, before proceeding to carry out their chemical identification. Such a method is doubtless serviceable in dealing with large bladder stones, but is difficult to apply to small calculi of renal and ureteral origin such as those which make up the bulk of specimens in a modern clinic. The method is not well adapted to the study of stones which contain intimate mixtures of certain constituents.

During the present century, analytical procedures have been improved and some of the elementary facts at the basis of stone formation have been discovered, so that Heller's scheme is no longer capable of satisfying present-day requirements. In recent years, a few articles have appeared, suggesting alternative procedures (4 to 9 ), some of which are quantitative (10 to 13 ). To judge the adequacy of these analytical schemes, we may set up the following criteria :

1. The tests used must be selected with regard to their simplicity and accuracy, and should be applicable to small amounts of material.

2. The scheme must be capable of analysing the mixtures which experience teaches us are likely to be found in naturally-occurring stones.

3. The results must be at least roughly quantitative, if the specimen is to be adequately classified.

The analytical schemes in the literature meet these criteria in varying degrees. Curiously enough, many of the authors, like Heller, make the assumption that they are dealing with a single constituent, a simplification which is far from warranted and which may obviously lead to erroneous conclusions. •
In the present scheme, the object has been to test for the presence of every common constituent of urinary stones, so that each will be identified if present in significant amounts. Where possible, the various constituents have been removed in consecutive steps, so that the chance of interference has been minimized. A sample of 5 to 10 mgm. is adequate, although it is generally possible to obtain a useful result from as little as 1 to 2 mgm.

The reagents are conveniently kept in $30 \mathrm{ml}$. dropping bottles with ground-in pipettes and nipples. The tests are carried out in $15 \mathrm{ml}$. centrifuge tubes, in volumes of 0.2 to $0.5 \mathrm{ml}$. In this way, consecutive separations may easily be made by use of the centrifuge.

\section{REAGENTS}

Hydrochloric Acid, Concentrated.

Nitric Acid, Concentrated.

Ammonium Hydroxide, Concentrated.

3 per cent Ammonium Molybdate.

Nessler's Reagent.

Sodium Acetate, Saturated Solution.

$2 M$ Ammonium Chloride-10.7 grams $\mathrm{NH}_{4} \mathrm{Cl}$ dissolved in $100 \mathrm{ml}$. of water.

$0.4 M$ Oxalic Acid-5.0 grams $\mathrm{C}_{2} \mathrm{H}_{2} \mathrm{O}_{4}, 2 \mathrm{H}_{2} \mathrm{O}$ in 100 ml. of water.

$1 M$ Disodium Hydrogen Phosphate-Dissolve 35.8 grams $\mathrm{Na}_{2} \mathrm{HPO}_{4}, 12 \mathrm{H}_{2} \mathrm{O}$ in $100 \mathrm{ml}$. of water.

$1 \mathrm{~N}$ Sodium Hydroxide.

5 per cent Sodium Cyanide.

Sodium Nitro-prusside Reagent. Dissolve 10 grams of the solid in $100 \mathrm{ml}$. of distilled water, adding $2 \mathrm{ml}$. of concentrated sulphuric acid.

Diazo Reagent. This must be freshly prepared by adding $0.3 \mathrm{ml}$. of 0.1 per cent Sodium Nitrite to $10 \mathrm{ml}$. of 0.1 per cent Sulphanilic Acid, which has been made up in a solution containing $15 \mathrm{ml}$. of concentrated Hydrochloric Acid per litre.

\section{PROCEDURE}

The stone is weighed, measured, and examined with the hand lens. Adherent blood is removed as far as possible. If necessary, the stone is sectioned to determine its homogeneity. One or more samples are taken, depending on the uniformity of the stone, and reduced to powder in a small mortar. 
1. Ignite some of the powder. Cystine, fat or wax, and blood clots will burn with a flame, if present in sufficient amounts. The purines glow without flame production. If no ash remains, it is unnecessary to carry out the tests for inorganic constituents, except for ammonia.

2. Uric acid. Every sample should be tested for the presence of uric acid. The well-known murexide test is satisfactory. One milligram or more of the powder is treated with 2 or 3 drops of concentrated $\mathrm{HNO}_{3}$ in a porcelain dish. The mixture is carefully evaporated to dryness, and heating continued until the colour change is complete. If the test is carried out on the water bath, uric acid gives first a yellow colour, which may be mistaken for the reaction for xanthine. If heating is continued a few minutes longer, xanthine shows no further alteration, but in the case of uric acid the colour changes to orange and finally to scarlet. On addition of a drop of ammonia, the uric acid oxidation product assumes a brilliant purple colour, while xanthine changes to orange, which becomes a red on further heating.

Since uric acid may occur as ammonium urate as well as the free acid, the test for ammonia should be made. The powder is extracted with hot dilute $\mathrm{HCl}$ and the extract tested for ammonia as in Step 9.

3. Xanthine. This substance may be identified as described in the previous paragraph. On account of its extreme rarity, a positive nitric acid test calls for confirmation. Ehrlich's diazo test may be used since uric acid is the commonest substance which might be mistaken for xanthine. A little of the powder is boiled with 1 to 2 $\mathrm{ml}$. of freshly-prepared diazo reagent, and a few drops of Normal $\mathrm{NaOH}$ are added. Xanthine couples with the reagant to produce a brilliant red wine colour. Uric acid gives no colour or a pale yellow, depending on the purity of the specimen. The test is not specific; it is given by many substances including the purines which are not substituted at positions 7 and 8 . Its use, however, assures that uric acid will not be mistaken for xanthine. The test might serve to detect xanthine in the presence of uric acid.

4. Cystine. A pinch of the powder is boiled with 2 to $3 \mathrm{ml}$. of water, then treated with an equal amount of 5 per cent $\mathrm{NaCN}$ solution. After 5 minutes, a few drops of sodium nitro-prusside solution are added. Cystine is indicated by a beautiful magenta colour. This test, described by Brand (14), is much quicker than the older method of recrystallizing from ammonia, and identifying the hexagonal crystals under the microscope. The solubility of the specimen in $\mathrm{NH}_{4} \mathrm{OH}$ and in dilute $\mathrm{HCl}$ should be tested as a rapid means of confirmation, and as a test of its purity, since no other common constituent of calculi is soluble in both. Cystine stones, as a rule, contain only small traces of other substances, and for ordinary purposes these do not need to be further identified.

5. Microscopic examination. This step is conveniently carried out at this time, in conjunction with Step 6. A little of the powder is mounted in water under a cover slip, and examined under the microscope. This gives added information as to the homogeneity of the speci- men, and may afford a clue as to its composition. The "triple phosphate" crystals of ammonium magnesium phosphate are sometimes easily recognized. Calcium oxalate may often be recognized under the hand lens as sharp, knife-edged crystals; when powdered, the crystals fracture irregularly, so that under the microscope the fragments resemble broken glass. Though calcium carbonate crystals may sometimes be recognized, other calcium salts are often amorphous or difficult to identify.

6. Test for carbonate. A little of the powder is placed on a glass slide and intimately mixed with a drop of water, to expel air bubbles. A cover slip, carrying a small drop of concentrated $\mathrm{HCl}$ is then inverted upon the powder. The evolution of $\mathrm{CO}_{2}$ can be readily recognized under the microscope.

Aside from this test for the identification of carbonate, which has been described by Newcomb (11), the behaviour of the powder after the addition of acid is often characteristic, and sufficient to indicate its composition. Phosphates and carbonates go rapidly into solution, oxalates dissolve slowly, while uric acid is insoluble. Cystine, on contact with concentrated $\mathrm{HCl}$, undergoes an instantaneous recrystallization which has been described by Wollaston (15).

7. Test for phosphate. A small amount-roughly 2 to $3 \mathrm{mgm}$. - of the powder is dissolved in 3 or 4 drops of concentrated $\mathrm{HNO}_{3}$, by boiling in a test tube. The solution is transferred to a centrifuge tube 1 and 3 or 4 drops of 3 per cent ammonium molybdate solution are added. A yellow precipitate indicates the presence of phosphate. This reaction may be slow, so that it is sometimes necessary to allow the tube to stand an hour or more before precipitation is complete. If proteins are present, they are thrown down by the reagent with formation of a white flocculent precipitate.

Experience has shown that tests carried out in $\mathrm{HNO}_{3}$ solution as described above are more sensitive than similar tests carried out in $\mathrm{HCl}$ solution. When dealing with stones which contain large amounts of phosphate it is more convenient to use a portion of the $\mathrm{HCl}$ solution prepared in Step 8. When small amounts of phosphate are to be detected, however, such as are often present in calcium oxalate stones, $\mathrm{HNO}_{3}$ solution is to be preferred, since $\mathrm{HCl}$ tends to increase the solubility of the phosphomolybdate.

8. Separation of acid-insoluble material. About 5 to $10 \mathrm{mgm}$. of the powder are boiled in a test tube with 1 to 2 drops of concentrated $\mathrm{HCl}$ and a little water, until the inorganic constituents have gone into solution. The solution is transferred to a centrifuge tube, diluted to about $0.7 \mathrm{ml}$, and insoluble residue is spun down. The supernatant fluid is removed with a dropping pipette and divided into two parts, or into three, if this solution is to be tested for phosphate. The insoluble residue, if any, is then examined under the microscope. It will usually consist of cellular and other protein debris. Uric acid may be present, as well as yellow-coloured hemo-

1 The powder may be dissolved by boiling in a Pyrex centrifuge tube, in order to avoid the necessity of transfer. 
globin derivatives. In stones from herbivorous animals, silica may be found.

If the supernatant is cloudy, it may be necessary to filter with suction through a small filter paper, about 1 $\mathrm{cm}$. in diameter, supported by a filter plate. The filter should be washed with a few drops of water to avoid loss of the solutes. The clear fluid obtained by centrifuging or filtration is known as Solution 1.

9. Test for ammonia. To part of Solution 1, a few drops of Nessler's solution are added, sufficient to make the mixture definitely alkaline. The formation of a white precipitate is disregarded. An orange colour or precipitate indicates the presence of ammonia. Formalin interferes with this test.

10. Test for calcium oxalate. To another part of Solution 1, add a few drops of saturated sodium acetate, until the acidity of the solution is brought to $\mathrm{pH} 5$ approximately, as indicated by Hydrion paper. 2 If calcium oxalate is present it will be precipitated almost quantitatively. The solution is mixed, permitted to stand for 15 minutes to allow complete precipitation, and the calcium oxalate thrown down in the centrifuge. The supernatant fluid (Solution 2) is transferred to another centrifuge tube.

This test for calcium oxalate is essentially that of Domanski (5). In our experience, the microscopic examination of the precipitate, and the permanganate test for confirmation are unnecessary refinements.

11. Test for non-oxalate calcium. To Solution 2, a drop of ammonium chloride is added, to prevent the precipitation of magnesium salts. Then 2 to 4 drops of oxalic acid solution are added, or sufficient to precipitate calcium which has not been precipitated in Step 10. Calcium, originally present as carbonate, phosphate, or citrate, is thrown down at this stage. The mixture is stirred, allowed to stand for 15 minutes, and centrifuged as before. The supernatant (Solution 3) is transferred to another centrifuge tube.

12. Test for magnesium. To Solution 3 , sufficient ammonium hydroxide is added to bring the $\mathrm{pH}$ to about 8 , by Hydrion paper. ${ }^{3}$ In order to make the reaction more sensitive, 1 or 2 drops of disodium hydrogen phosphate are also added. A crystalline precipitate indicates the presence of magnesium. The star-shaped crystals which form should be checked by microscopic examination. Carried out in this way, the test is sufficiently sensitive to detect the magnesium present in a small sample of bone.

2 Those who prefer the use of a liquid indicator may add a drop of 0.016 per cent solution of brom cresol green, then add the acetate solution until the color changes from yellow to green. In order to avoid increasing the volume it may be advantageous to place the drop on a white porcelain tile and use it as an external indicator.

${ }^{3} \mathrm{~A} 0.5$ per cent solution of phenolphthalein in 50 per cent alcohol may be used as an indicator for this step, if preferred.

\section{DISCUSSION}

The method described above has certain novel features. Among these is the separation of the calcium into oxalate and non-oxalate fractions. Here the question of the qualitative detection of non-oxalate calcium is not the essential point, as experience has shown that it is present to some extent in practically every inorganic stone. On the other hand, it is sometimes important to have some idea of the relative amounts of these calcium fractions. If the separation is made carefully, in a semi-quantitative way, the volumes of the precipitates may be compared after centrifuging. One can then decide by inspection whether one is dealing with a stone which is predominantly composed of calcium oxalate, or whether oxalate is present in traces in a stone of fundamentally different nature.

A more important feature of the scheme is the determination of the presence or absence of magnesium. Strangely enough, it has been customary to neglect this step in qualitative examination of calculi. For instance, Domanski (5) omits the differentiation between phosphates, and states that if phosphate is present it is legitimate to assume the presence of calcium and magnesium phosphate. In our experience, this is not the case. There is an important group of phosphate stones which do not contain magnesium, as will be shown in the following paper.

Finally, if we allow ourselves to make certain assumptions which are commonly made about the composition of inorganic stones, the method permits certain checks on the accuracy of the results.

Thus, if magnesium is absent, carbonate or phosphate should be accompanied by non-oxalate calcium in at least equivalent amounts. Similarly, since ammonia probably means either ammonium urate or ammonium magnesium phosphate, if the former is absent the ammonia should correspond to the magnesium, at least in the case of stones caused by urea-splitting organisms. Experience tends to show that these rules are qualitatively valid, in an approximate way, for human material.

During the four years in which the method has been in routine use in this laboratory, no difficulties have arisen in its application. It is necessary to caution a technician using it for the first time against the use of too much of the powdered stone 
for the analysis, or using the concentrated reagents too freely, as salts may be formed in amounts exceeding their solubilities and so give rise to unexpected precipitates.

One might imagine cystine being mistaken for calcium oxalate in Steps 8 and 10, as it would go into solution in dilute acid, and unless a very small specimen were taken, might precipitate on addition of sodium acetate. In such case, no confusion should arise if ignition has been carried out beforehand in proper sequence. Wollaston's crystals will also be recognized in Step 6. In actual work, no difficulty has been experienced on this score.

A more serious problem is the identification of citrate, which has been reported in experimental rat calculi, but has not been found in significant amounts in human stones (16). There does not seem to be any test for the qualitative identification of citrate, suitable for routine use in the analysis of urinary calculi.

\section{SUMMARY}

1. A method for the qualitative chemical examination of urinary calculi is described.

2. The distinctive features of the method include the separation of the calcium into oxalate and non-oxalate fractions, and the determination of the presence or absence of magnesium.

\section{BIBLIOGRAPHY}

1. Heller, J. F., Die Harnconcretionen, ihre Entstehung, Erkennung, und Analyse mit besonderer Rücksicht auf Diagnose und Therapie der Nieren- und Blasenerkrankung. Wein, Tendler u. Comp., 1860.

2. Randall, A., Campbell, E. W., and Beeson, H. C., A simple method of chemical analysis of urinary calculi with a report of the chemical composition of a recent series. Urol. and Cutan. Rev., 1934, $38,29$.

3. Keyser, L. D., Recurrent urolithiasis : etiologic factors and clinical management. J. A. M. A., 1935, 104, 1299.

4. Ranganathan, S., Chemical composition of urinary calculi in rats. Indian J. M. Research, 1930, 18, 599.

5. Domanski, T. J., Renal calculi : a new method for qualitative analysis. J. Urol., 1937, 37, 399.

6. Domanski, T. J., Analysis of urinary calculi. Am. J. Clin. Path., Tech. Suppl., 1938, 2, 157.

7. Kamlet, J., Analysis of renal calculi. J. Lab. and Clin. Med., 1937, 23, 321.

8. Higgins, C. C., and Mendenhall, E. E., Factors associated with recurrent formation of renal lithiasis with report of new method for qualitative analysis of urinary calculi. J. Urol., 1939, 42, 436.

9. Seifter, J., and Trattner, H. R., Simplified qualitative analysis of urinary calculi by spot tests. J. Urol., 1939, 42, 452.

10. Newcomb, C., and McCarrison, R., The composition of vesical calculi. Indian J. M. Research, 1928-29, 16, 1036.

11. Newcomb, C., A scheme for the analysis of small urinary calculi. Indian J. M. Research, 1930, 17, 735.

12. Kya, L. T., The composition of vesical calculi. Chinese M. J., 1936, 50, 797.

13. Brown, H., Micromethods for the quantitative analysis of urinary calculi. J. Lab. and Clin. Med., 1939, 24, 976.

14. Brand, E., Harris, M. H., and Biloon, S., Cystinuria. The excretion of a cystine complex which decomposes in the urine with the liberation of free cystine. J. Biol. Chem., 1930, 86, 315.

15. Wollaston, W. H., On cystic oxide, a new species of urinary calculus. Proc. Roy. Soc., London, 1810, 100, 223.

16. Schneider, H., and Steenbock, H., Calcium citrate uroliths on a low phosphorus diet. J. Urol., 1940, 43, 339. 